\title{
Protective Effect of Biobran/MGN-3 against Sporadic Alzheimer's Disease Mouse Model: Possible Role of Oxidative Stress and Apoptotic Pathways
}

\author{
Mamdooh H. Ghoneum ${ }^{1}{ }^{1}$ and Nesrine S. El Sayed $\mathbb{D}^{2}$ \\ ${ }^{1}$ Department of Surgery, Charles Drew University of Medicine and Science, Los Angeles, California, USA \\ ${ }^{2}$ Department of Pharmacology and Toxicology, Faculty of Pharmacy, Cairo University, Cairo, Egypt \\ Correspondence should be addressed to Nesrine S. El Sayed; nesrine_salah2002@yahoo.com
}

Received 28 September 2020; Revised 14 December 2020; Accepted 3 January 2021; Published 27 January 2021

Academic Editor: Kambiz Hassanzadeh

Copyright (c) 2021 Mamdooh H. Ghoneum and Nesrine S. El Sayed. This is an open access article distributed under the Creative Commons Attribution License, which permits unrestricted use, distribution, and reproduction in any medium, provided the original work is properly cited.

\begin{abstract}
Alzheimer's disease (AD) is a debilitating and irreversible brain disease that affects an increasing number of aged individuals, mandating the development of protective nutraceuticals. Biobran/MGN-3, an arabinoxylan from rice bran, has potent antioxidant, antiaging, and immunomodulatory effects. The aim of the present study was to investigate the protective effect of Biobran against sporadic Alzheimer's disease (SAD). SAD was induced in mice via intracerebroventricular injection of streptozotocin (STZ) $(3 \mathrm{mg} / \mathrm{kg})$. STZ-treated mice were administered with Biobran for 21 days. The effects of Biobran on memory and learning were measured via the Morris water maze, novel object recognition, and Y-maze tests. Biomarkers for apoptosis, oxidative stress, and amyloidogenesis were measured using ELISA and western blot analysis. Histopathological examination was performed to confirm neuronal damage and amyloid-beta deposition. Biobran reversed the spatial memory deficit in SAD-induced mice, and it increased the expression of glutathione, reduced malondialdehyde, decreased IL-6, decreased intercellular adhesion molecule-1 (ICAM-1), and significantly increased nuclear factor erythroid 2-related factor 2 (Nrf2) and antioxidant response element (ARE). Moreover, Biobran exerted a protective effect against amyloid-beta-induced apoptosis via the suppression of both cleaved caspase- 3 and the proapoptotic protein Bax and via the upregulation of the antiapoptotic protein Bcl-2. Furthermore, it reduced the expression of forkhead box class O proteins. It could be concluded from this study that Biobran may be a useful nutritional antioxidant agent for protection against SAD through its activation of the gene expression of Nrf2/ARE, which in turn modulates the apoptotic and amyloidogenic pathways.
\end{abstract}

\section{Introduction}

Alzheimer's disease (AD) is the most common neurodegenerative disorder characterized by progressive loss of memory and cognition. The appearance of oxidative stress markers is one of the hallmarks of $\mathrm{AD}$; it leads to the build-up of amyloid deposits and neurofibrillary tangles and to the progression of the disease [1]. Oxidative stress is involved in many disorders, including Parkinson's disease, chronic inflammation, and $\mathrm{AD}$ [2]. Neurons produce energy at a high rate and show high oxygen consumption; they are at extremely high risk to oxidative damage from reactive oxygen species (ROS) [3]. Currently, the process by which amyloid-beta $(\mathrm{A} \beta)$ accumulation occurs in the central nervous system is uncertain, but the generation of ROS during $A \beta$ selfaggregation is a potential mechanism by which $\mathrm{A} \beta$ may cause neuronal damage and death. This effect ultimately leads to synaptic membrane depolarization, excessive calcium influx, and mitochondrial impairment $[4,5]$. One of the common regulators of the oxidative stress pathway in $\mathrm{AD}$ is the expression of the nuclear factor erythroid 2-related factor 2 (Nrf2). It is present mainly in the cytoplasm of the 
hippocampal neurons [6], and in AD animal models, the pathology of $\mathrm{A} \beta$ is linked with altered expression of $\mathrm{Nrf} 2$ target genes [7]. Nrf2 can act as a molecular switch in neurons that mediates the antioxidant system [8]. Active Nrf2 protects cells against oxidative injury by binding to the antioxidant response element (ARE) under oxidative stimuli and promoting antioxidative genes [9]. Therefore, the restoration of Nrf2 expression could alleviate cognitive impairment by protecting neurons against oxidative injury and decreasing $\mathrm{A} \beta$ accumulation [10].

Neuroinflammation plays an important role in $\mathrm{AD}$ pathogenesis through the production of inflammatory cytokines like IL-6, which is highly prevalent in $\operatorname{AD}[11,12]$, and the intercellular adhesion molecule-1 (ICAM-1), which is highly expressed in the neuritic plaques in $\mathrm{AD}$ brains. ICAM-1 has been implicated in neurodegeneration through its role as an important mediator of immune cell activation and inflammatory response in AD [13]. ICAM-1 plays a key role in cell survival in the brain and induced the upregulation of proapoptotic proteins, Bax and cleaved caspase-3, and downregulation of the antiapoptotic proteins, Bcl-2 $[14,15]$. This pathway has many downstream targets like the transcriptional factor forkhead box proteins of class O3a (FOXO3a), a factor that, when translocated to the nucleus, can trigger cell apoptosis. Evidence is growing that apoptotic markers can directly target FOXO3a and lead to cell apoptosis [16]. The family of FOXO proteins is extensively involved in the cell signal transduction of apoptosis and in oxidative stress. This effect is important in the survival of cerebral endothelial vascular cells [17], oxidative stress injury in mouse cerebellar granule neurons [18], and hippocampal neuronal injury [19]; and it can also lead to caspase 3induced apoptotic death $[20,21]$.

STZ injection in the brain is linked to brain insulin resistance, neuroinflammation, oxidative stress, and deposition of $\mathrm{A} \beta$, as well as tau protein aggregation leading to impairment in memory and learning functions that mimic sporadic Alzheimer's disease (SAD) in humans [22]. ICV-STZ injection induces the activation of microglial cells, which produce massive amounts of inflammatory mediators and free radicals that provoke neuronal damage [23].

Currently, there are no dietary supplements or prescribed medications for decreasing the risk of AD [24], and current FDA-approved treatments for $\mathrm{AD}$ are only symptomatic [25]. Biobran/MGN-3 is a denatured hemicellulose from rice bran that has shown promising effect as a natural adjuvant to existing immunotherapies for cancer [26, 27] through its antioxidant properties [28]. Biobran was shown previously to enhance natural killer cell activity in aged mice [29] as well as healthy elderly human subjects [30], with improvement in their health-related quality of life [31]. Few studies have been done on the beneficial effects of Biobran on aging and neurodegenerative diseases. So, the present study is aimed at investigating the possible protective effect of Biobran in a SAD model through the modulation of oxidative stress, amyloidogenesis, inflammation, and apoptotic pathways. Behavioral and biochemical experiments were performed to illuminate the mechanisms underlying its potential neuroprotective effect in the STZ model of SAD.

\section{Material and Methods}

2.1. Animals. Adult male Swiss albino mice, weighing 25$30 \mathrm{~g}$, were used in the present study. Mice were obtained from the animal facility of the National Research Center, Cairo, Egypt, and they were housed 6 mice per cage. Mice were allowed to acclimate to their environment for a period of one week prior to the study. Animals were maintained in a controlled environment with constant temperatures $\left(25 \pm 2^{\circ} \mathrm{C}\right)$, light/dark cycles $(12 / 12 \mathrm{~h})$, and relative humidity $(60 \pm 10 \%)$. Animals were provided with a standard chow diet and allowed water ad libitum. This study complied with the Guide for the Care and Use of Laboratory Animals published by the US National Institutes of Health [32] and was approved by the Institutional Animal Care and Use Committee, Cairo University (CU-IACUC), approval number: CUIII-F-26-20. Animal discomfort and suffering was minimized as much as possible.

2.2. Drug Treatments. STZ was purchased from SigmaAldrich Co. (St Louis, MO, USA) and dissolved in saline solution $(0.9 \% \mathrm{NaCl})$. Biobran is a denatured hemicellulose that is extracted from rice bran by reacting rice bran hemicellulose with carbohydrate-hydrolyzing enzymes obtained from Shiitake mushrooms. Biobran's main chemical structure is arabinoxylan with an arabinose polymer in its side chain and a xylose in its main chain [26]. Daiwa Pharmaceutical Co. Ltd. (Tokyo, Japan) kindly provided Biobran. Biobran was prepared in saline $(0.9 \% w / v)$ and was freshly prepared each day (Figure 1).

2.3. Acute Toxicity Study. Earlier studies have shown Biobran to be a nontoxic and safe agent. The Ames mutagenicity test is negative, Biobran's 50\% lethal dose (LD50) is greater than $36 \mathrm{~g} / \mathrm{kg}$, and several toxicity studies have all confirmed the safety of Biobran in both humans and animals [27, 33]. 8month-long periods of treatment of animals with Biobran as well as 5-year-long treatment for humans [34] have not resulted in any adverse side effects. In the present study, Biobran's acute toxicity in mice was assessed via the up and down procedure according to Guideline No. 423 from the Organization for Economic Cooperation and Development [35]. A starting dose of $2 \mathrm{~g} / \mathrm{kg}$ Biobran was given to mice. For 24 hours, the mice were observed continuously for toxic symptoms, following which they were observed daily over an additional 20 days of maintenance.

2.4. Induction of $S A D$. SAD was induced in mice by ICV injection of STZ $(3 \mathrm{mg} / \mathrm{kg})$ into their lateral ventricle using the freehand procedure [36] updated by Warnock [37] for the avoidance of cerebral vein penetration. Thiopental ( $50 \mathrm{mg} / \mathrm{kg}$, i.p.) was used to anesthetize the mice. The mouse head was secured using downward pressure above the ears, followed by insertion of the needle directly through the skin and skull into the lateral ventricle. Visualization of an equilateral triangle between the eyes and center of the skull was used to locate the bregma and target the lateral ventricle allowing the needle to be inserted at the following coordinates from bregma: $1 \mathrm{~mm}$ mediolateral, $0.1 \mathrm{~mm}$ anteroposterior, 


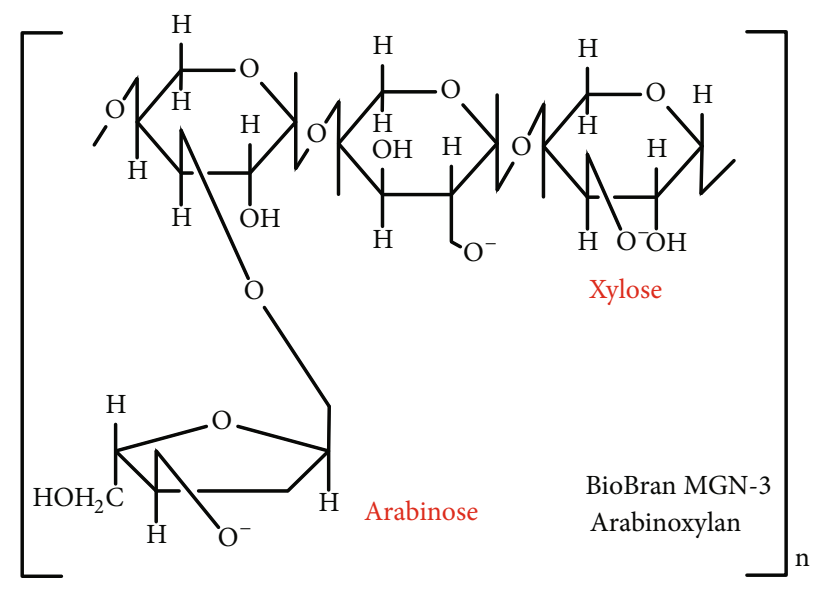

Figure 1: Chemical structure of Biobran/MGN-3.

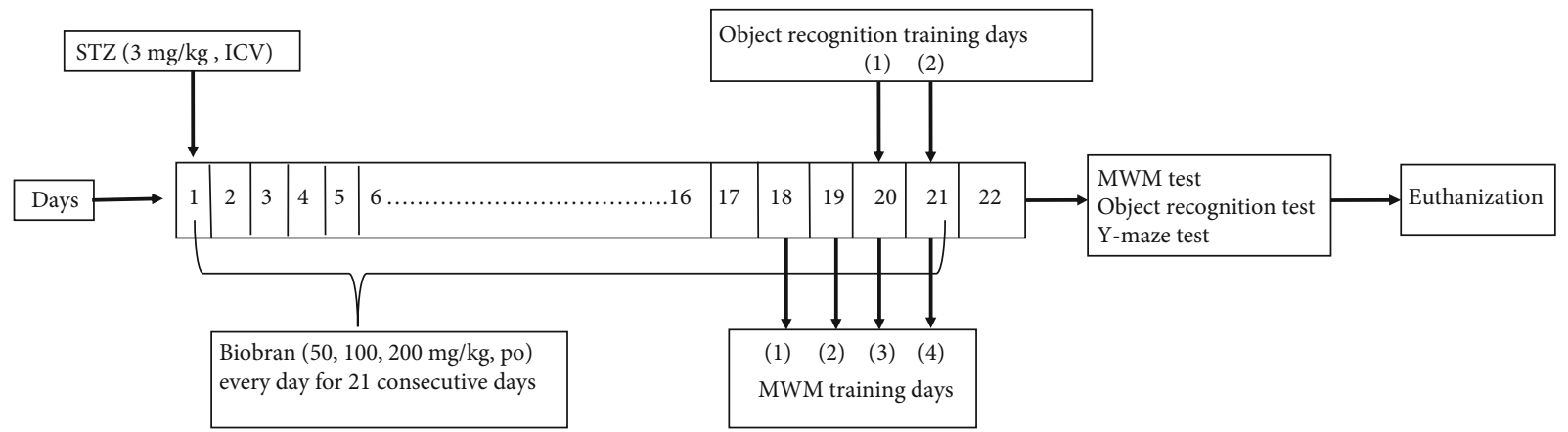

FIgURE 2: Experimental design.

and $3 \mathrm{~mm}$ dorsoventral. The mice exhibited normal behavior approximately 1 minute after injection.

2.5. Experimental Design. Mice were randomly allocated into five groups, each containing 12 mice. Group 1, the sham control group, received one ICV saline injection followed by intraperitoneal (i.p.) saline injections for 21 consecutive days. Group 2 received STZ ( $3 \mathrm{mg} / \mathrm{kg}$, ICV) once and served as the SAD model [38]. Group 3 received STZ ( $3 \mathrm{mg} / \mathrm{kg}$, ICV), five hours later, it is followed by Biobran ( $50 \mathrm{mg} / \mathrm{kg}$, i.p.) daily for 21 consecutive days. Group 4 received STZ (3 mg/kg, ICV), five hours later, it is followed by Biobran $(100 \mathrm{mg} / \mathrm{kg}$, i.p.) daily for 21 consecutive days. Group 5 received STZ $(3 \mathrm{mg} / \mathrm{kg}$, ICV), five hours later, it is followed by Biobran $(200 \mathrm{mg} / \mathrm{kg}$, i.p.) daily for 21 consecutive days. Once the treatment period ended, mice were given behavioral tests assessing cognitive functions (Figure 2).

\subsection{Behavioral Assessments}

2.6.1. Object Recognition Test. To assess long-term memory and estimate cognition, we used the object recognition test. It is based on the concept of preference for novelty, which is the innate tendency of animals to exhibit an affinity for exploring a novel object rather than a familiar one [39]. This test was administered over three consecutive days. On day 1 , each mouse was placed in a wooden box $(30 \times 30 \times 30)$ dimension and was left for thirty minutes to adapt to the surroundings.

On day 2, two wooden cubes identical in shape, color, and size were placed at opposite corners in the box, $2 \mathrm{~cm}$ from the walls. Each mouse was placed in the box's middle and given ten minutes to explore the new objects. On day 3, one of the cubes was replaced by a novel object of different shape, size and color, and mice were each given five minutes to explore the box's objects. Objects and the arena were thoroughly cleaned with $70 \%$ ethanol between experiments with individual mice to ensure that their behavior was not guided by odor cues. Each mouse was faced toward the same wall at the beginning of each trial and was prevented from displacing the objects. Animals were video-recorded and measured for the following:

(1) Discrimination Index. Time difference between the exploration of novel and familiar objects divided by the total exploration time

(2) Recognition Index. Time spent exploring the novel object as a percentage of the total exploration time

2.6.2. Morris Water Maze (MWM) Test. The MWM test was used to investigate spatial memory and learning [40]. The maze consisted of stainless-steel circular tanks $(210 \mathrm{~cm}$ in diameter, $51 \mathrm{~cm}$ high) filled with water $\left(25 \pm 2^{\circ} \mathrm{C}\right)$ to a depth of $35 \mathrm{~cm}$ and divided into four quadrants. A black platform 
(10 cm wide, $28 \mathrm{~cm}$ high) was placed inside the target quadrant and submerged by $2 \mathrm{~cm}$. The platform remained in the same location during training and testing procedures, and it was made invisible by coloring the water with a purplecolored nontoxic dye. Memory-acquisition trials (120 s/trail) were performed over four consecutive days, twice/day, with at least $15 \mathrm{~min}$ between trials. Animals were left free during each acquisition trial to locate the hidden platform. If the mouse located the platform, it was given 20 additional seconds for rest, while if the mouse did not reach the platform within $120 \mathrm{~s}$, it was guided to the platform and allowed to rest there for $20 \mathrm{~s}$. Mean escape latency (MEL) was calculated as the time taken by each mouse to find the hidden platform. After four acquisition trial days, mice were allowed $60 \mathrm{sec}-$ onds to probe a pool in which the platform had been removed. Mice were put into the water in the Northeast position (Q4), and this is a fixed release point during the test. Measurements were made of the time each mouse spent in the target quadrant as an indicator of retrieval or memory.

2.6.3. Y-Maze Test. The Y-maze is used to measure spatial working memory in rodents via the spontaneous alternation behavior (SAB) calculation [41]. Spontaneous alternation measures the ability of the animal to alternate its choice of arm entry on subsequent trials based on its memory of previous arm entries performed, which depends on the natural exploratory behavior of animals for new environments. The maze is a Y-shaped apparatus consisting of three arms, each one with the same dimensions, $35 \mathrm{~cm}$ long, $25 \mathrm{~cm}$ high, and $10 \mathrm{~cm}$ wide at $120^{\circ}$ extending from a central platform. The apparatus was placed on the floor of the experimental room. The test was performed for 2 days. The first day was for the purpose of training; each mouse was positioned in the central platform and allowed to explore the maze freely for 8 minutes. The same procedure was followed on the second testing day, with the addition of manual recording of each arm entry, scored only when all four limbs of the mouse were inside the arm. After each session, the maze was cleaned with $70 \%$ ethanol to exclude any olfactory cues that might interfere with subsequent testing. An alternation was considered to have occurred if three successive different arms were entered during an overlapping triplet set. The percentage of spontaneous alternation activity (SAB\%) was calculated as the "number of alternations consecutively" divided by "the total number of arm entries minus 2" and multiplied by 100 .

2.7. Biochemical Assessments. Following the behavioral tests, animals $(n=12)$ were anesthetized using thiopental sodium ( $50 \mathrm{mg} / \mathrm{kg}$, i.p.) and then euthanized by cervical dislocation. The brains were rapidly dissected on ice/salt mixture and washed with ice-cold saline. The hippocampi were homogenized in ice-cold saline to prepare $10 \%$ homogenates; these were split into several aliquots and stored at $-80^{\circ} \mathrm{C}$ for estimation of the biochemical parameters $(n=6)$, western blot analysis $(n=3)$, and histopathological examination $(n=3)$.

\subsection{Biochemical Measurements}

2.8.1. Determination of GSH and MDA. The hippocampal glutathione (GSH) content was measured spectrophotometri- cally using Ellman's reagent [42]. The peroxidation of hippocampal lipids was estimated by measuring malondialdehyde (MDA) levels via thiobarbituric acid reactive substances [43]. The results are expressed as $\mathrm{mmol} / \mathrm{mg}$ protein.

2.8.2. Determination of IL-6, ICAM-1, Cleaved Caspase-3, and Amyloid- $\beta_{1-42}$. Hippocampal IL-6 and ICAM-1 levels were estimated using mouse ELISA kits purchased from RayBiotech Inc. (Norcross, Georgia, USA) and MyBioSource Inc. (San Diego, CA, USA), respectively. Cleaved caspase-3 and amyloid- $\beta_{1-42}$ mouse ELISA kits were provided from Cusabio, Wuhan, China. The hippocampal levels of these markers were measured according to the manufacturer's instructions for each respective ELISA kit and expressed as their corresponding units to the tissue protein content determined by Salama et al. [44]. The ELISA assay measures the amount of sample by sandwiching it between two antibodies, one of which is precoated to the microtiter plate, and the other of which acts as a detector antibody. The microtiter plate provided in each kit was precoated with an antibody specific to each marker. Standards or samples are then added to the appropriate microtiter plate wells with a biotinconjugated antibody preparation specific for the marker, and avidin conjugated to HRP is added to each microplate well and incubated. Then, a TMB substrate solution is added to each well. Only those wells that contain the protein, biotinconjugated antibody, and enzyme-conjugated avidin will exhibit a change in color. The enzyme-substrate reaction is terminated by the addition of a sulfuric acid solution, and the color change is measured spectrophotometrically at a wavelength of $450 \mathrm{~nm}$.

2.8.3. Western Blot Analysis. Western blot is a method to quantify the expression level of specific proteins. Protein solutions were extracted from hippocampal tissues, $n=3$. SDS-PAGE (10\% acrylamide gel) was used to separate equal amounts of protein (averaging 20-30 $\mu \mathrm{g}$ of total protein). Proteins were subsequently transferred to polyvinylidene difluoride membranes (Pierce, Rockford, IL, USA) with a Bio-Rad Trans-Blot system. Western blot immunodetection was performed by incubating the membranes at room temperature for 1 hour with blocking solution comprised of $20 \mathrm{mM}$ Tris-Cl, pH 7.5, $150 \mathrm{mM} \mathrm{NaCl}, 0.1 \%$ Tween 20, and $3 \%$ bovine serum albumin. Membranes were incubated overnight at $4^{\circ} \mathrm{C}$ with one of the following primary antibodies: Nrf2 (catalog no: 31163), FOXO3a (catalog no: 1950), and $\beta$-actin (catalog no: 8227) obtained from Thermo Fisher Scientific Inc. (Rockford, IL, USA). Peroxidase-labelled secondary antibodies (1:1000; Novus Biologicals, Colorado, USA) were added after washing, followed by $1 \mathrm{~h}$ of membrane incubation at room temperature. Incubation with the substrate permits the detection of the amount of protein through optical documentation systems. The quantification of the band intensity can be used to determine specific protein levels in the tested tissues. Detection of a second "housekeeping" protein is necessary to control for variability in protein loading between samples. The band intensity was analyzed using the ChemiDoc ${ }^{\mathrm{TM}}$ imaging system with Image LabTM software version 5.1 (Bio-Rad Laboratories Inc., Hercules, CA, 


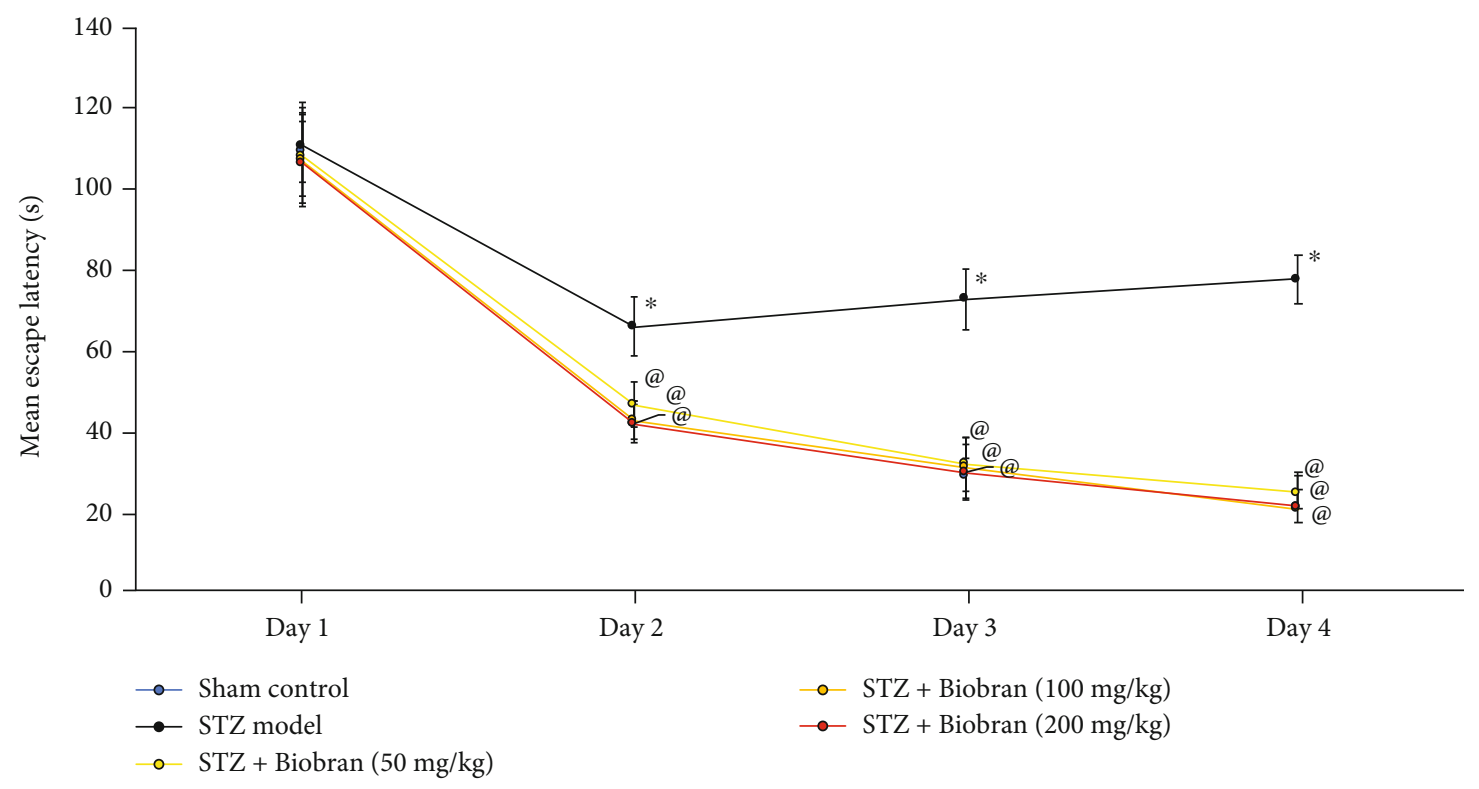

(a)

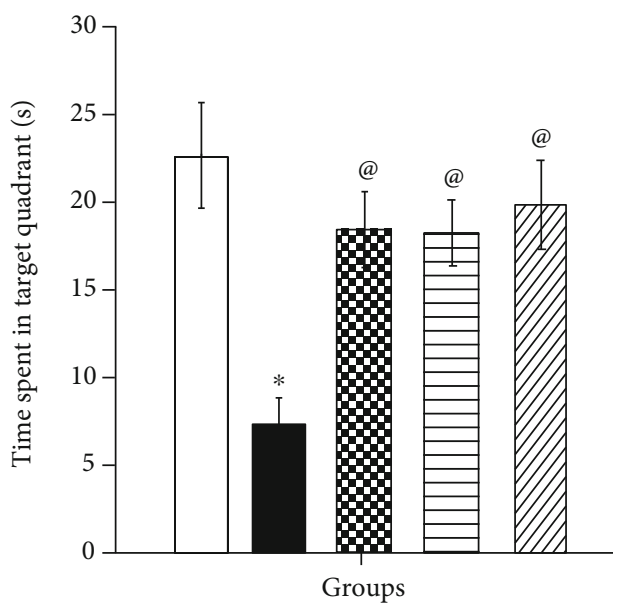

(b)

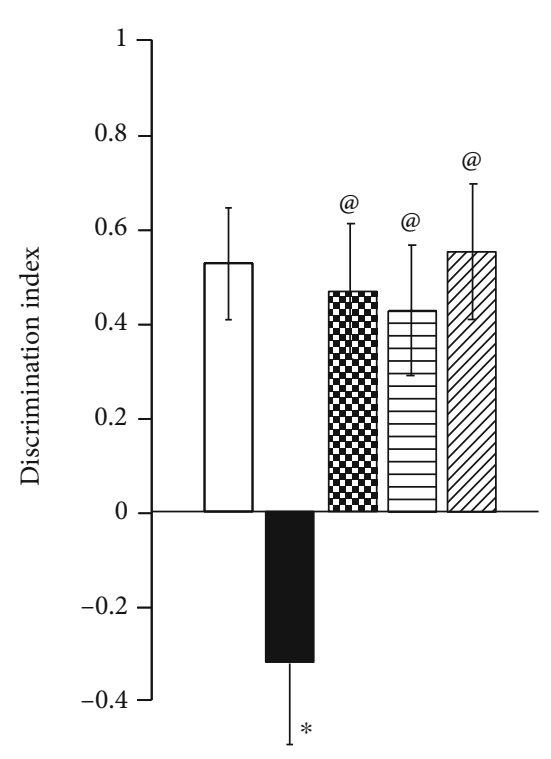

Groups

(c)

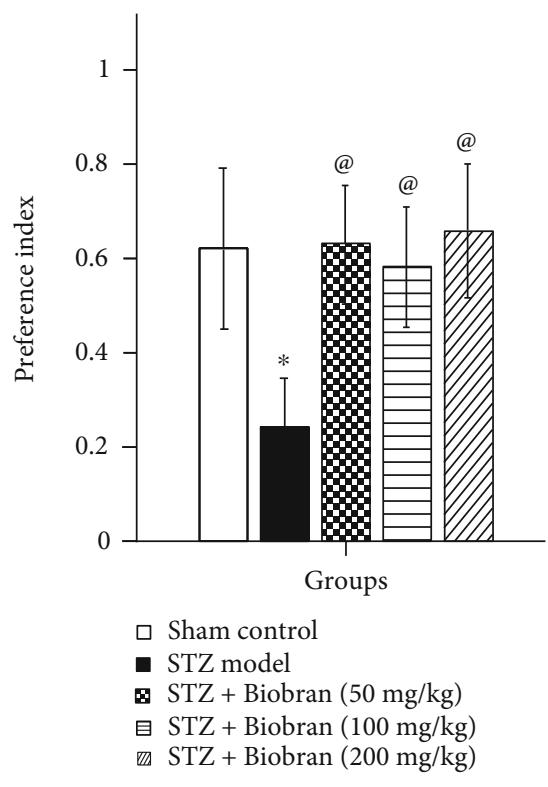

(d)

FIGURE 3: Effect of Biobran on the cognitive functions in MWM and NOR tasks in ICV-STZ injected mice. (a) Effect of Biobran on MEL in MWM. (b) Effect of Biobran on time spent in target quadrant in MWM. (c) Effect of Biobran on the discrimination index in NOR. (d) Effect of Biobran on the preference index in NOR. Values are expressed as mean \pm SD; $n=12$. Statistical analyses were performed using one-way analysis of variance (ANOVA) followed by the Tukey-Kramer post hoc test. ${ }^{*}$ Significantly different from normal group at $p<0.05$. ${ }^{\circledR}$ Significantly different from ICV-STZ group at $p<0.05$.

USA). The results are presented in arbitrary units after normalization to levels of the $\beta$-actin protein.

\subsubsection{Histopathological Analysis of Brain}

(1) $H \& E$ Staining. The brain of 3 mice in each group was excised and fixed in $10 \%$ formol saline for $24 \mathrm{~h}$. Washing was done in tap water then serial dilutions of alcohol (methyl, ethyl, and absolute ethyl) were used for dehydration. Specimens were cleared in xylene and embedded in paraffin at $56^{\circ} \mathrm{C}$ in a hot air oven for $24 \mathrm{~h}$. Paraffin bees wax tissue blocks were prepared for sectioning at $4 \mu \mathrm{m}$ thickness by sledge microtome. The obtained tissue sections were collected on glass slides, deparaffinized, and stained by hematoxylin \& eosin (H\&E) stain for examination using a light electric microscope [45]. 
TABLE 1: Effect of Biobran (50, 100, and $200 \mathrm{mg} / \mathrm{kg}$ ) on spontaneous alternation behavior in Y-maze task in ICV-STZ-injected mice. Values are expressed as mean $\pm \mathrm{SD} ; n=12$. Statistical analyses were performed using one-way analysis of variance (ANOVA) followed by the Tukey-Kramer post hoc test. ${ }^{*}$ Significantly different from the normal group at $P<0.05$, ${ }^{@}$ Significantly different from the ICV-STZ group at $P<0.05$.

\begin{tabular}{|c|c|c|c|c|c|}
\hline Groups & $\begin{array}{l}\text { Sham } \\
\text { control }\end{array}$ & STZ model & $\begin{array}{l}\text { STZ + Biobran } \\
\quad(50 \mathrm{mg} / \mathrm{kg})\end{array}$ & $\begin{array}{l}\text { STZ + Biobran } \\
(100 \mathrm{mg} / \mathrm{kg})\end{array}$ & $\begin{array}{c}\text { STZ + Biobran } \\
(200 \mathrm{mg} / \mathrm{kg})\end{array}$ \\
\hline $\begin{array}{l}\text { \% Spontaneous } \\
\text { alternation }\end{array}$ & $68.29 \pm 2.07$ & ${ }^{*} 35.72 \pm 1.72$ & ${ }^{\circledR} 55.79 \pm 1.55$ & ${ }^{\circledR} 59.32 \pm 1.68$ & ${ }^{\circledR} 65.87 \pm 2.05$ \\
\hline
\end{tabular}

(2) Congo Red Staining. For Congo red staining, sections were stained with Congo red solution $(0.2 \%)$ for $1 \mathrm{~h}$ and then counter-stained with hematoxylin solution. Plaques were observed and captured at $400 \mathrm{X}$ magnification under a fluorescent microscope.

2.9. Statistical Analysis. Data are presented as mean \pm S.D. Mean escape latency in Morris water maze trials was analyzed by repeated-measures analysis of variance (ANOVA). The remaining results were analyzed using one-way ANOVA followed by Tukey's multiple comparison test. Statistical analysis was performed using GraphPad Prism(c) software (version 6.01; Graph Pad Software, California, USA). For all the statistical tests, the level of significance was fixed at $P<0.05$.

\section{Results}

3.1. Biobran's acute toxicity was investigated. We found no mortality, toxicity, or general behavior changes over a $24 \mathrm{~h}$ period at a dose of $2 \mathrm{~g} / \mathrm{kg}$

3.2. Neurobehavioral Analysis. The effects of STZ and Biobran $(50,100$, and $200 \mathrm{mg} / \mathrm{kg}$ ) on neurobehavioral tests were conducted within $24 \mathrm{~h}$ of the last day of Biobran injection.

3.2.1. Mean Escape Latency (MEL). Figure 3(a) shows that the MEL in the MWM for STZ-treated mice was significantly higher $(159 \%)$ than the MEL of the sham control mice at day 2 , an effect that further increased at day 3 and 4 . Mice treated with Biobran, on the other hand, had MEL values that were similar to the sham control starting on day 2 .

3.2.2. Time Spent in the Target Quadrant. Studies of Biobran's effect on time spent in the target quadrant of the MWM revealed that STZ-treated mice spent $32.6 \%$ of the time in the quadrant in comparison with the sham control, while animals treated with 50,100, and $200 \mathrm{mg} / \mathrm{kg}$ of Biobran spent $81.7 \%, 81.0 \%$, and $88.1 \%$ of the time, respectively, as compared to STZ-treated mice $[F(4,55)=92.80$, $P<0.0001$ ] (Figure 3(b)).

3.2.3. Discrimination and Preference Indices in the Novel Object Recognition (NOR) Test. The NOR test is used to examine the effect of STZ and Biobran on discrimination and preference indices. Administration of STZ in mice resulted in a decrease of the discrimination index compared to sham-control mice; on the other hand, it was increased significantly after Biobran administration in a dose-dependent manner. Additionally, the time ICV-STZ injected mice spent exploring the novel object was 39\% of the time of the sham control group, which reflects a lower preference index. On the other hand, mice supplemented with Biobran were observed to prefer the novel object over the familiar object, normalizing the preference index in a dose-dependent manner (Figures 3(c) and 3(d)).

3.2.4. Spontaneous Alternation Behavior in Y-Maze Task. The ICV-STZ group exhibited a significantly lower percentage of spontaneous alteration behavior, as compared to sham control mice. Treatment with different doses of Biobran resulted in a significant increase in the percentage of spontaneous alteration behavior, as compared to the ICV-STZ group $[F(4,55)=37.90, P<0.0001]$. Thus, Biobran attenuated the STZ-induced impairment in short term memory as its administration caused a significant elevation in the percentage of spontaneous alteration behavior (Table 1).

3.3. Oxidative Stress Biomarkers. The levels of MDA and GSH in the hippocampus were measured to investigate Biobran's protective effect on oxidative stress biomarkers. Administration of STZ resulted in a significant decrease of the GSH level by $15.5 \%$ compared to the sham control mice However, the administration of Biobran resulted in a significant increase in GSH content in a dose-dependent manner that was maximized at $82.8 \%$ at $200 \mathrm{mg} / \mathrm{kg}$ compared to ICV-STZ-injected mice $[F(4,40)=771.4, P<0.0001]$. On the other hand, intracerebroventricular injection of STZ produced a significant increase in the MDA levels compared to sham control mice. Biobran supplementation for SAD mice reduced the MDA level in a dose-dependent manner compared to STZ-injected mice $[F(4,40)=2180, P<0.0001]$. Moreover, Biobran $(200 \mathrm{mg} / \mathrm{kg})$ decreased significantly the MDA level as compared to Biobran (50 mg/kg) (Figure 4).

Oxidative stress was further studied via the expression of Nrf2 and ARE. Mice treated with STZ exhibited a significant decrease in hippocampal Nrf2 and ARE levels as compared to sham control mice, while exposure to Biobran resulted in a dose-dependent reversal of the expression of Nrf2 and ARE as compared to STZ-injected mice $[F(4,40)=285.5, P<$ $0.0001],[F(4,40)=455.5, P<0.0001]$, respectively, where a high dose of Biobran $(200 \mathrm{mg})$ approximately returned Nrf2 and ARE levels to that of control (Figure 5).

3.4. Amyloid $\beta_{1-42}$. STZ-treated mice exhibited approximately a 4-fold increase in $\mathrm{A} \beta$ expression in comparison with the sham control. The $A \beta$ levels were significantly decreased in STZ-treated mice after administration of Biobran to STZ injected mice $[F(4,40)=2348, P<0.0001]$. The effect was 


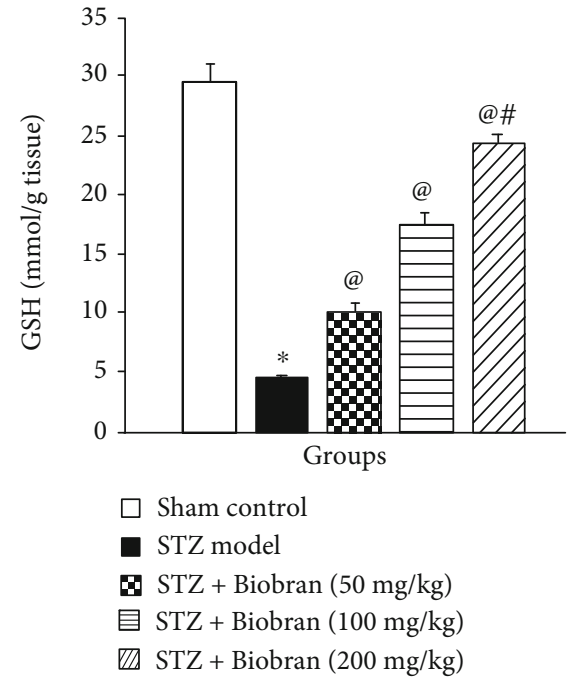

(a)

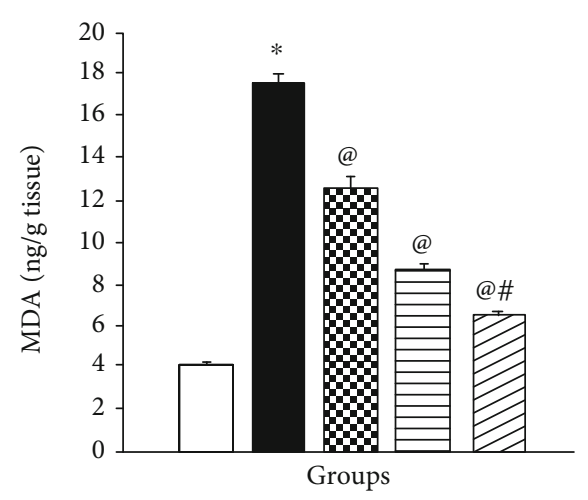

$$
\begin{aligned}
& \square \text { Sham control } \\
& \text { STZ model } \\
& \text { STZ + Biobran }(50 \mathrm{mg} / \mathrm{kg}) \\
& \text { 目 } \mathrm{STZ}+\text { Biobran }(100 \mathrm{mg} / \mathrm{kg}) \\
& \text { 四 } \mathrm{STZ}+\text { Biobran }(200 \mathrm{mg} / \mathrm{kg})
\end{aligned}
$$

(b)

Figure 4: Effect of Biobran on MDA and GSH in ICV-STZ-injected mice. Values are expressed as mean \pm SD; $n=6$. Statistical analyses were performed using one-way analysis of variance (ANOVA) followed by the Tukey-Kramer post hoc test. *Significantly different from normal

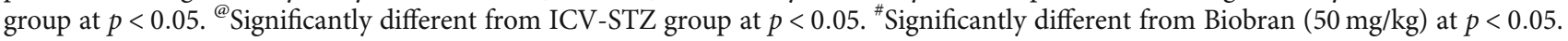
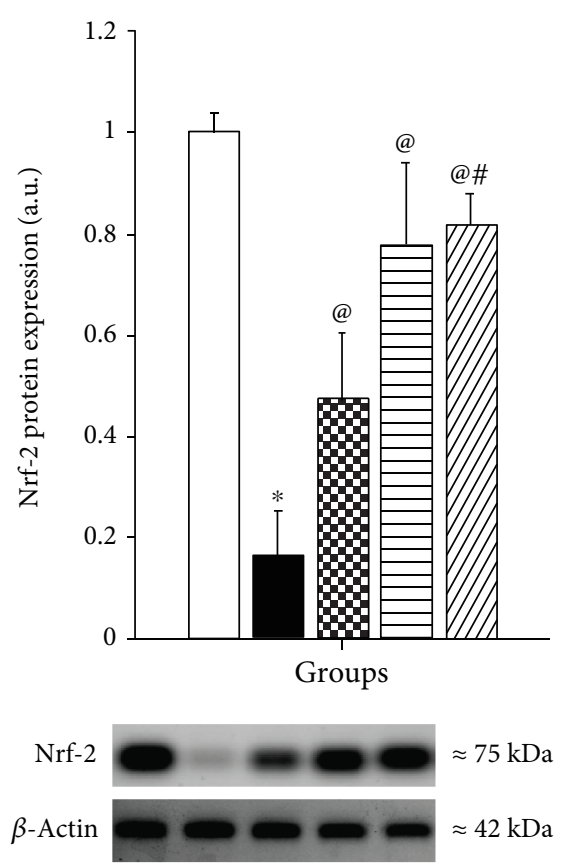
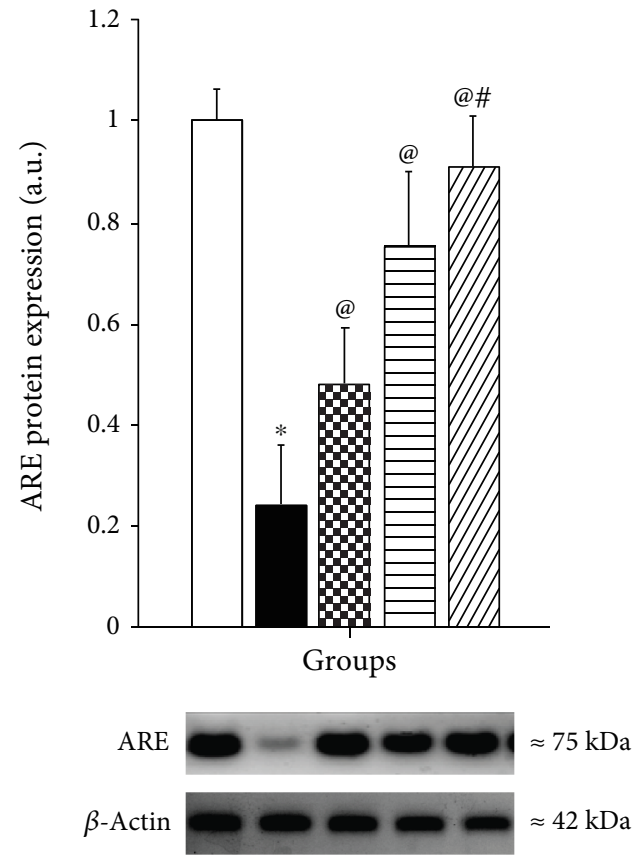

目 STZ + Biobran $(100 \mathrm{mg} / \mathrm{kg})$

四 STZ + Biobran $(200 \mathrm{mg} / \mathrm{kg})$

Figure 5: The effect of Biobran on Nrf2 and ARE in ICV-STZ-injected mice. Values are expressed as mean \pm SD; $n=3$. Statistical analyses were performed using one-way analysis of variance (ANOVA) followed by the Tukey-Kramer post hoc test. * Significantly different from normal group at $p<0.05$. ${ }^{@}$ Significantly different from ICV-STZ group at $p<0.05$. " Significantly different from Biobran $(50 \mathrm{mg} / \mathrm{kg})$ at $p<$ 0.05 .

dose-dependent, reaching the lowest level for $200 \mathrm{mg} / \mathrm{kg}$ (Figure 6).

3.5. Inflammatory Biomarkers. In $\mathrm{AD}$ and many other diseases, autoimmune and inflammatory processes can be stim- ulated by IL-6. STZ-treated mice had a significant increase in IL-6 and ICAM-1 expression as compared to control mice. However, Biobran supplementation suppressed the levels of these markers in a dose-dependent manner that reached the control level at $200 \mathrm{mg} / \mathrm{kg}$ as compared to ICV-STZ mice 


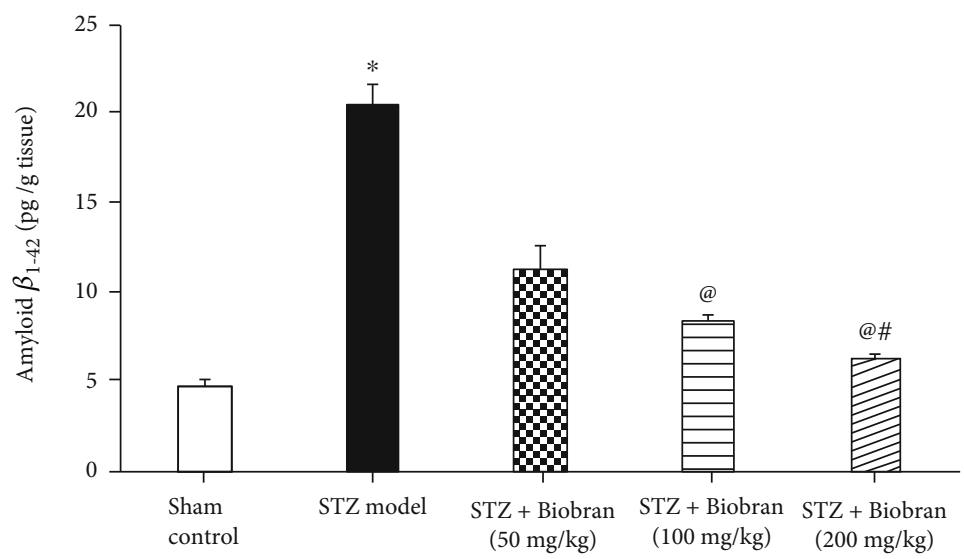

Figure 6: The effect of Biobran on $\mathrm{A} \beta_{1-42}$ in ICV-STZ-injected mice. Values are expressed as mean \pm SD; $n=6$. Statistical analyses were performed using one-way analysis of variance (ANOVA) followed by the Tukey-Kramer post hoc test, *Significantly different from normal group at $p<0.05$. ${ }^{\circledR}$ Significantly different from ICV-STZ group at $p<0.05$. " Significantly different from Biobran $(50 \mathrm{mg} / \mathrm{kg})$ at $p<0.05$.

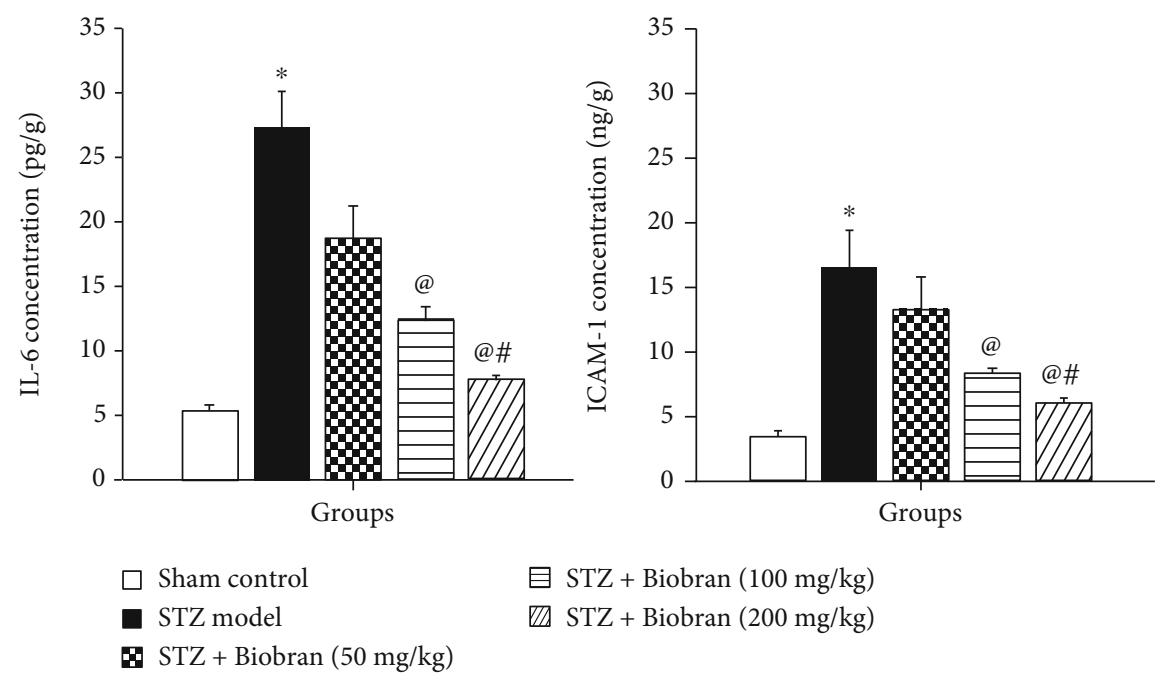

Figure 7: Effect of Biobran on IL-6 and ICAM-1 in ICV-STZ-injected mice. Values are expressed as mean \pm SD; $n=6$. Statistical analyses were performed using one-way analysis of variance (ANOVA) followed by the Tukey-Kramer post hoc test. *Significantly different from normal group at $p<0.05$. ${ }^{\circledR}$ Significantly different from ICV-STZ group at $p<0.05$. " Significantly different from Biobran (50 mg/kg) at $p<0.05$.

$[F(4,40)=646.2, P<0.0001]$ and $[F(4,40)=857.1, P<$ $0.0001]$, respectively (Figure 7).

3.6. Apoptotic Biomarkers. STZ-treated mice exerted a significantly increased expression of Bax while simultaneously showing a decrease in $\mathrm{Bcl}-2$ expression in comparison to sham control. The Bax/Bcl-2 ratio in STZ-treated mice is 38 times greater compared to the ratio for sham control mice. In contrast, mice supplemented with Biobran demonstrated a dose-dependent reversal of Bax and Bcl-2 expression relative to STZ-treated mice. At the highest Biobran concentration, both Bax and Bcl-2 expression were comparable to the sham control level, with the $\mathrm{Bax} / \mathrm{Bcl}-2$ ratio being only 1.5 times as high as the sham control (Figure 8(a)). A similar effect was seen for cleaved caspase- 3 expression. In STZ mice, cleaved caspase- 3 levels increased approximately 5 -fold relative to control. Follow- ing supplementation with Biobran, STZ-treated mice exhibited a gradual decrease in cleaved caspase- 3 expression in a dose-dependent manner $[F(4,40)=1728, P<$ 0.0001] (Figure 8(b)).

We furthermore analyzed the expression of FOXO3a protein. A significant increase by 5-fold in the expression of FOXO3a was revealed in STZ-treated mice relative to control. Following supplementation with Biobran, FOXO3a expression in STZ-treated mice decreased in a dosedependent manner, with the highest dose $(200 \mathrm{mg})$ nearly bringing FOXO3a expression back to the level of control compared to STZ-injected mice $[F(4,25)=670.3, P<$ 0.0001], respectively] (Figure 9).

3.7. Histopathology Analysis. Brains of sham control mice showed normal structure of the brain tissue including the cerebral cortex and the hippocampus. Microscopic 


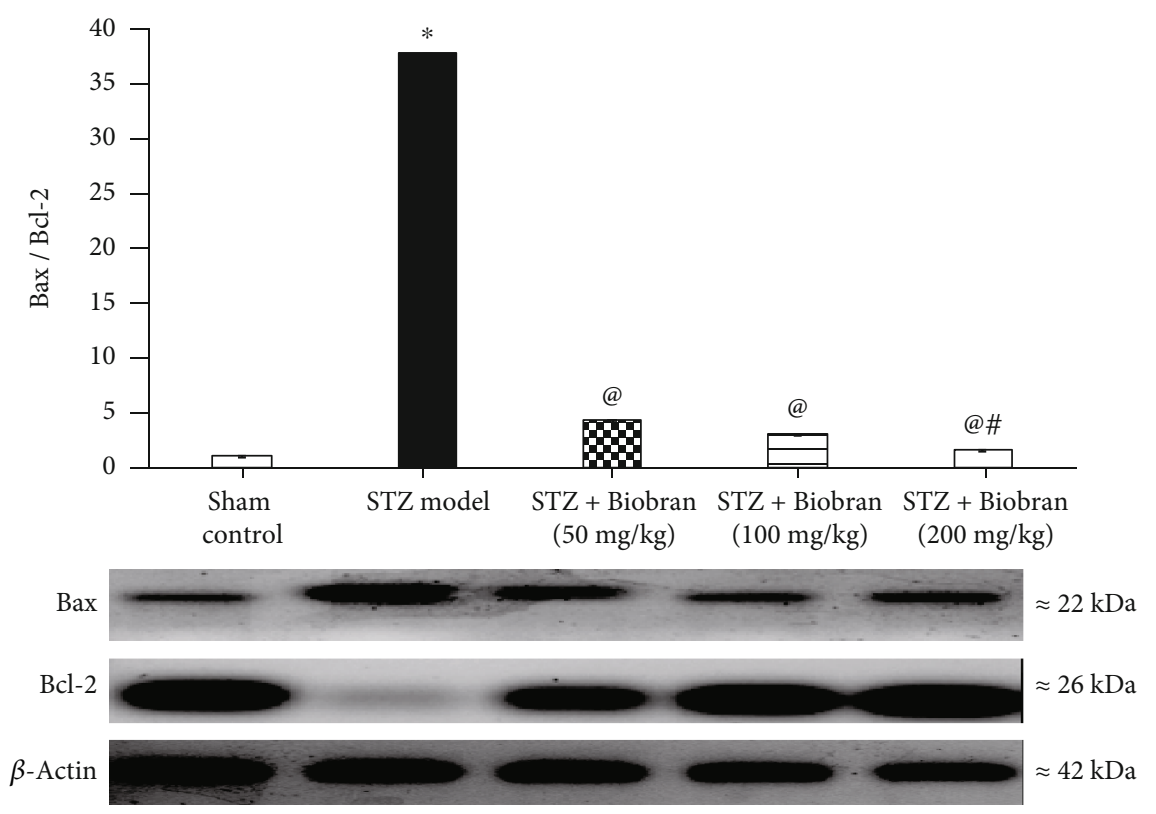

(a)

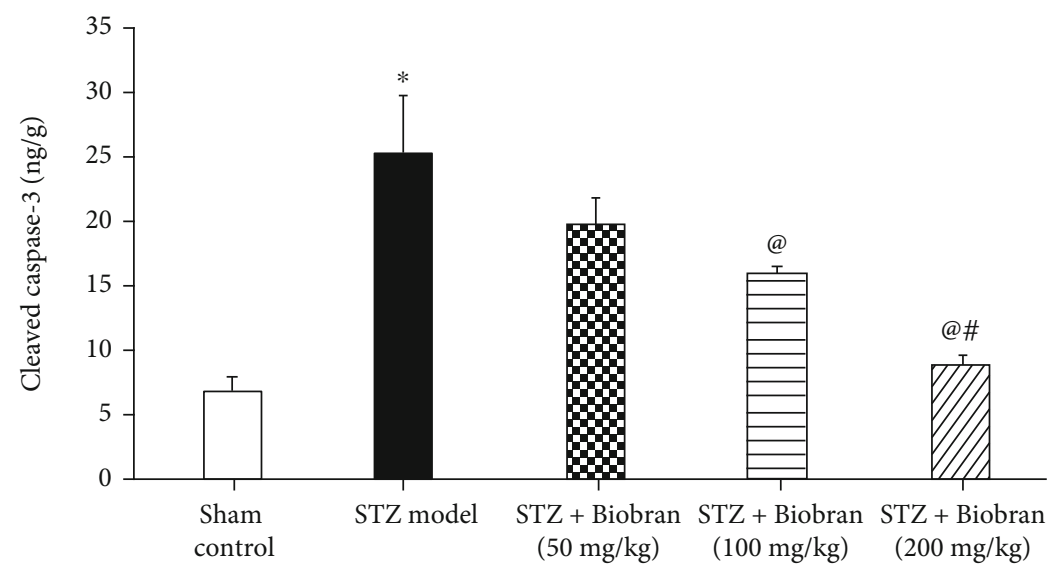

(b)

FIgURE 8: Effect of Biobran on the ratio of Bax to Bcl-2 (a) and cleaved Caspase-3 (b) in ICV-STZ-injected mice. Values are expressed as mean \pm SD; $n=3$. Statistical analyses were performed using one-way analysis of variance (ANOVA) followed by the Tukey-Kramer post hoc test. ${ }^{*}$ Significantly different from normal group at $p<0.05$. ${ }^{@}$ Significantly different from ICV-STZ group at $p<0.05$. ${ }^{*}$ Significantly different from Biobran $(50 \mathrm{mg} / \mathrm{kg})$ at $p<0.05$.

examination of the STZ group showed several histopathological changes in the brain tissue. The cerebral cortex showed numerous scattered dark degenerated neurons that were associated with neuronophagia and diffuse gliosis. The hippocampus showed multifocal haemorrhagic areas with dark degenerated neurons in the CA3, CA4, and DG regions.

Mice treated with Biobran $(50 \mathrm{mg} / \mathrm{kg})$ ameliorated the effects of STZ. Sections of the cerebral cortex showed decreased numbers of dark neurons with healthy neurons in most examined sections. The hippocampus showed normal neurons in the various neurological regions.

Mice treated with Biobran $(100 \mathrm{mg} / \mathrm{kg}$ and $200 \mathrm{mg} / \mathrm{kg}$ ) showed normal histological structure of the cerebral cortex except for few degenerated neurons and neuronophagia. The hippocampus appeared apparently normal.
The administration of Biobran showed no histopathological alterations in the brain tissue with normal structure of the cerebral cortex and the hippocampus (Figures 10 and 11).

The number of amyloid plaques was investigated in different experimental groups through visualization with Congo red stain. Normal mice showed no amyloid deposition in the brain sections. Meanwhile, ICV injection of STZ showed multifocal deposition of the amyloid deposition in the brain tissue especially in the inflammatory lesion that showed focal gliosis. The administration of Biobran $(50 \mathrm{mg} / \mathrm{kg})$ resulted in a marked reduction of the number of amyloid plaques in the brain tissue. Moreover, mice that received Biobran $(100 \mathrm{mg} / \mathrm{kg})$ showed few amyloid plaques, and mice that received Biobran $(200 \mathrm{mg} / \mathrm{kg})$ showed an absence of amyloid plaques in most examined brain tissue (Figure 12). 

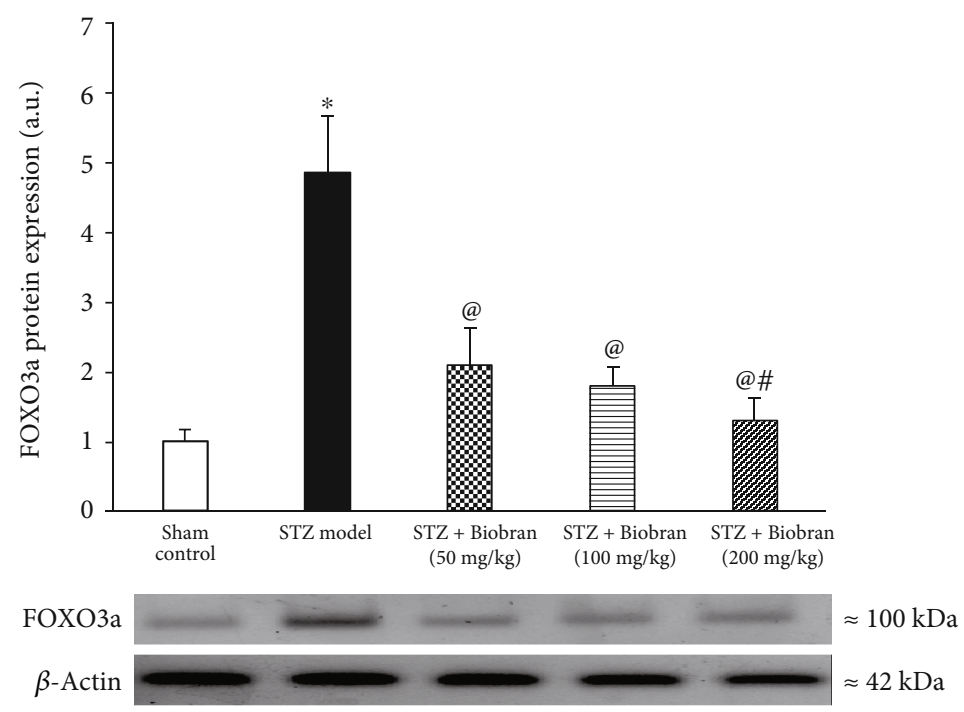

FIGURE 9: Effect of Biobran on FOXO3a in ICV-STZ injected mice. Values are expressed as mean \pm SD; $n=3$. Statistical analyses were performed using one-way analysis of variance (ANOVA) followed by the Tukey-Kramer post hoc test. * Significantly different from normal group at $p<0.05$. ${ }^{\circledR}$ Significantly different from ICV-STZ group at $p<0.05$. " Significantly different from Biobran (50 mg/kg) at $p<0.05$.

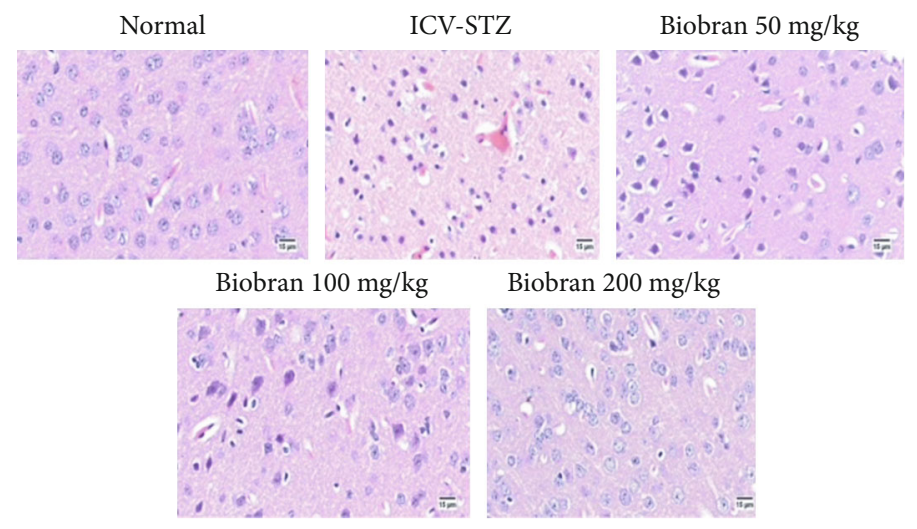

Figure 10: Effect of Biobran (50, 100, and $200 \mathrm{mg} / \mathrm{kg}$ ) on histopathological changes in the cerebral cortex of ICV-STZ injected mice, $n=3$. Control: showing normal histological structure of the cerebral cortex of normal mice; ICV-STZ: showing diffuse gliosis in the cerebral cortex admixed with numerous degenerated neurons in the injected mice; Biobran $50 \mathrm{mg} / \mathrm{kg}$ : showing moderate number of degenerated neurons; Biobran 100 mg/kg: showing few injured neurons; Biobran $200 \mathrm{mg} / \mathrm{kg}$ : showing apparently normal cerebral cortex structure.

\section{Discussion}

The current study evaluated the protective effect of Biobran/MGN-3 against STZ-induced SAD in mice. Biobran, a natural biological response modifier, has been shown to possess antiaging [29-31] and antioxidant [37] properties. Biobran is proved previously to exhibit potent immunomodulatory functions [26, 27, 46-48] and exert beneficial effects against cancer, viruses, and microbes [49-52].

In the present study, STZ-treated mice were unable to discriminate between novel and familiar objects, as demonstrated by the NOR task. The ICV-STZ group revealed marked deterioration in memory and learning functions as observed in the Morris water maze and manifested by a significant decrease in the time spent in the target quadrant as well as in the Y-maze tests demonstrated by a significant decline in the spontaneous alternation behavior. These findings are in agreement with previous studies reporting that ICV-STZ injection was implicated in decreased spontaneous alternation behavior in the Y-maze test and a decline in spatial learning and reference memory in Morris water maze trials as well as the test day $[53,54]$. This indicates obvious memory and learning deficits in these mice. The ICV injection of STZ is a well-known model of sporadic Alzheimer's disease in rodents with similar progressive pathology of $\mathrm{AD}$ as in the human brain [55-57]. However, it was of great interest to note that Biobran supplementation prevented the STZ-induced impairments of short-term and spatial memory. In a dose-dependent manner, Biobran reduced the MEL time, extended the time spent in the target quadrant, and reversed the discrimination and preference indices as well as decreasing the spontaneous alternation behavior in the Y-maze task.

Coherent to the aforementioned findings, it was found that the cognitive dysfunction exerted a positive impact on 


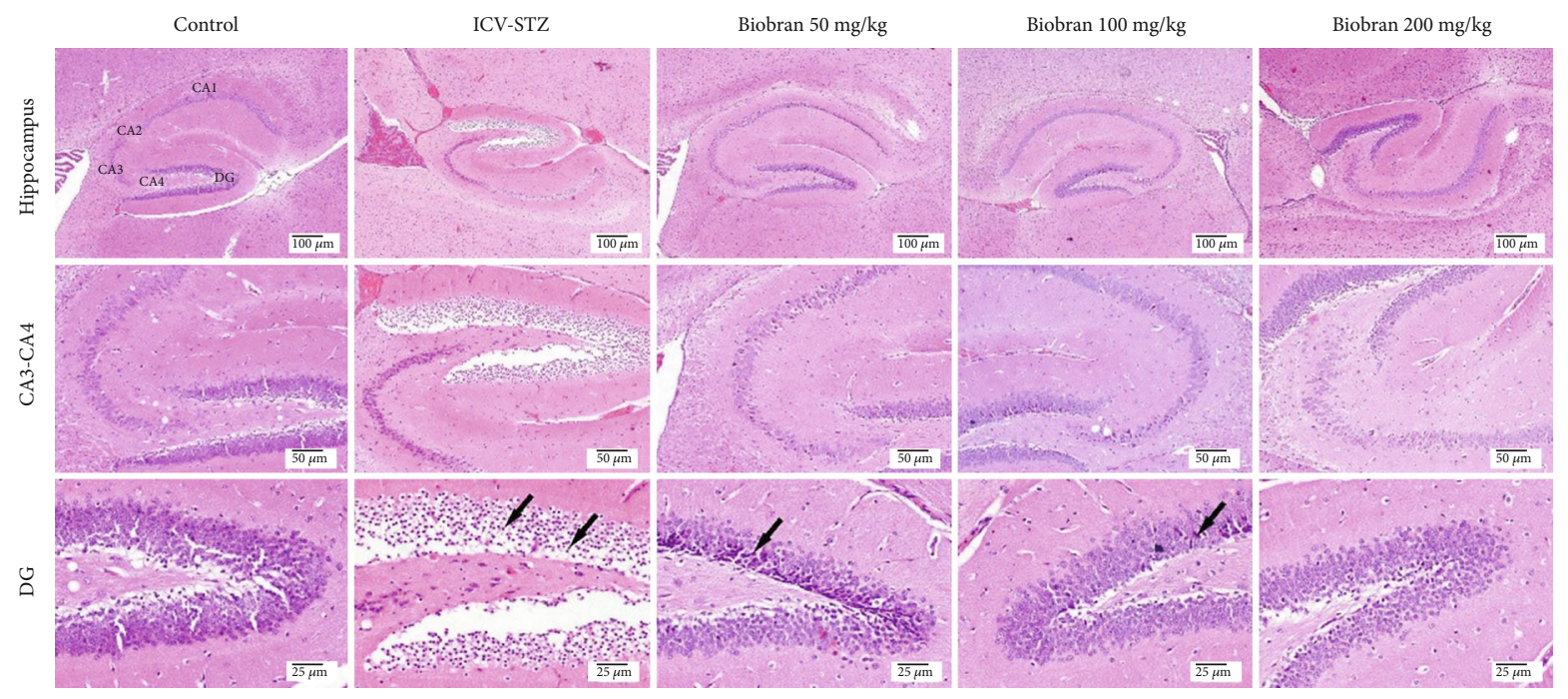

Figure 11: Effect of Biobran (50, 100, and $200 \mathrm{mg} / \mathrm{kg}$ ) on histopathological changes in the hippocampus of ICV-STZ-injected mice, $n=3$. Control: showing normal histological structure of different CA regions and DG of the hippocampus of normal mice; ICV-STZ: showing edema of CA1, CA2, and DG with dark-degenerated neurons in CA3, CA4, and DG (arrows) in the hippocampus and congestion in the surrounding brain parenchyma; Biobran $50 \mathrm{mg} / \mathrm{Kg}$ : showing dark degenerated neurons in the CA1, CA4, and DG regions (arrow) of the hippocampus; Biobran $100 \mathrm{mg} / \mathrm{Kg}$ : showing apparently normal neurons in the hippocampus with few scattered neurons in CA4 and DG (arrow); Biobran $200 \mathrm{mg} / \mathrm{Kg}$ : showing apparently normal neurons in the hippocampus.

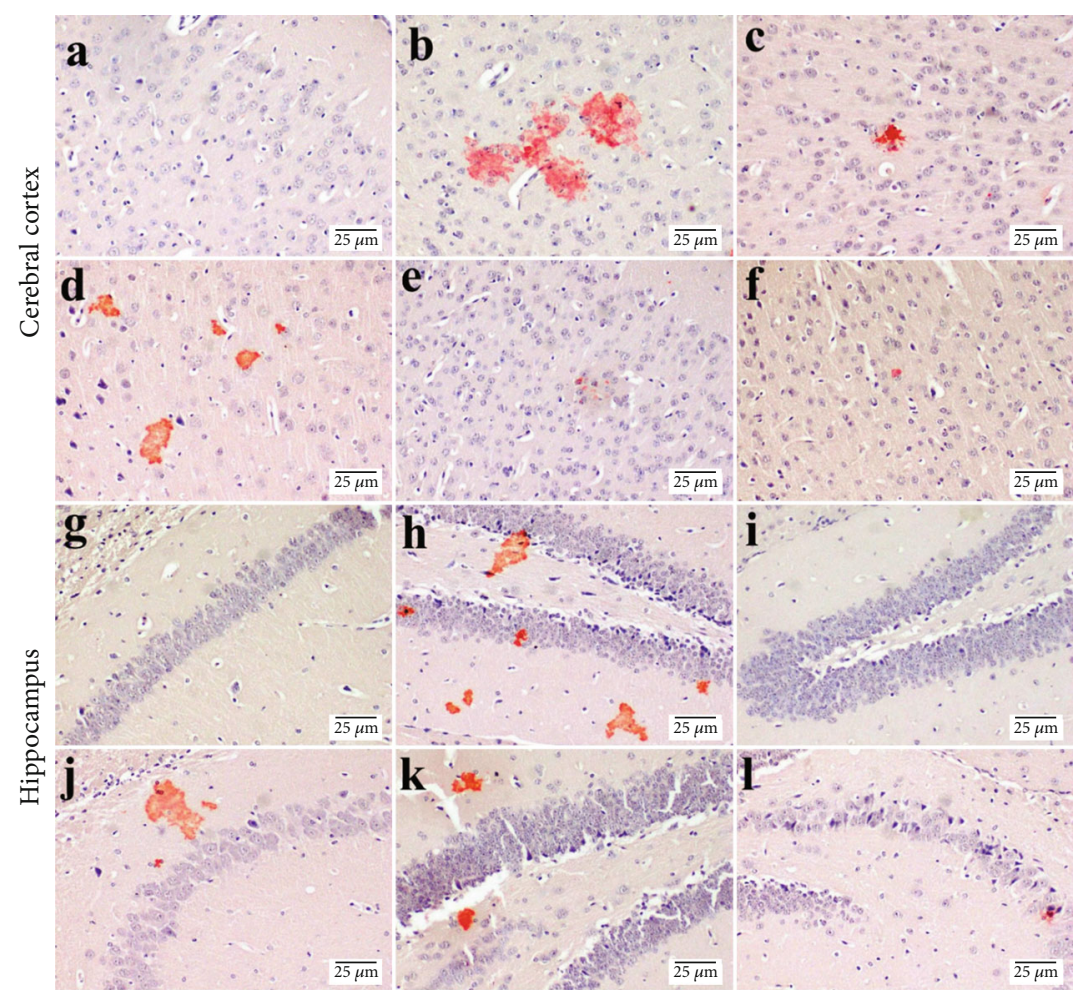

FIGURE 12: Congo red-brain-stained sections of mice for amyloid plaques visualization in the cerebral cortex and hippocampus $(n=3)$. (a, g, i) represent the normal group showing no deposition of amyloid plaques. (b, h) represent the STZ group showing multifocal deposition in the cerebral cortex in the cerebral cortex and the hippocampus, respectively. (c, d, j) represent Biobran (50 mg/kg) in STZ-injected mice, showing multifocal scattered plaques. (e, k) represent Biobran $(100 \mathrm{mg} / \mathrm{kg})$ in STZ-injected mice, showing few deposition in the cerebral cortex and multifocal deposition in the hippocampus. (f, l) represent Biobran $(200 \mathrm{mg} / \mathrm{kg}$ ) in STZ-injected mice showing minute deposition of amyloid plaques. 
the amyloidogenic and oxidative stress pathways. $A \beta$ peptide is one of the hallmarks of $\mathrm{AD}$ causing neuronal loss in the brain and resulting into deficits in memory and learning. Previous studies revealed that antioxidant compounds could be promising therapeutic or preventive interventions for $\mathrm{AD}$ patients because they inhibit $\mathrm{A} \beta$ fibril formation and protect the brain from $\mathrm{A} \beta$ neurotoxicity [58]. In the current study, Biobran exerted a significant antioxidant effect in the model of SAD, which is in agreement with previous studies that revealed Biobran's antioxidant activity against murine solid Ehrlich carcinoma [28], as well as its ability to significantly alleviate the increase in MDA content and prevent the irradiation-induced depletion of GSH in mice spleens [59].

ROS generation caused by mitochondrial oxidative phosphorylation can have profound effects on cellular functions and result in the initiation of many diseases, including aging $[60]$ and AD $[2,61]$. During oxidative stress, ROS can lead to neuronal synaptic dysfunction $[62,63]$ and may cause neuronal damage and death during $\mathrm{A} \beta$ self-aggregation [64]. Biobran has been shown previously to upregulate the oxidative stress in the liver and to inhibit the levels of these biomarkers including MDA, total free radicals, and nitric oxide in murine Ehrlich carcinoma [28]. This suggests that Biobran induces oncostatic activity by providing protection against oxidative stress, modulating lipid peroxidation, and enhancing the antioxidant defense system. Moreover, it was reported previously that Nrf2 is suppressed in AD patients' neurons [65], which is in harmony with the results of the present study. For AD animals, there is a decrease in Nrf2 expression, as well as in the expression of the Nrf2/ARE pathway's target genes [66]. Altered expression in Nrf2 is associated with cognitive deficits and impaired spatial memory in mouse models of $\mathrm{AD}$ [6] and a deficiency in Nrf2 results in vulnerability to oxidative stress [67], phosphorylated-Tau [68], and enhanced autophagic dysfunction [7]. On the other hand, it was revealed previously that neurons can be protected against $\mathrm{A} \beta$ pathology and oxidative proteotoxic stress by the upregulation of the Nrf2/ARE pathway $[69,70]$. Several studies have shown that neuropathological changes such as $\mathrm{AD}$ and Parkinson's are also associated with faulty inflammatory processes such as increased expression of the proinflammatory cytokine IL-6 in the brain $[11,12]$. In the current study, IL-6 and ICAM-1 were significantly increased after STZ administration, but Biobran supplementation caused a significant decrease in the levels of these biomarkers. Interestingly, a recent clinical study revealed the increased concentrations of IL- 6 and ICAM- 1 in AD patients' cerebrospinal fluid (CSF) [71, 72]. These effects were more evident in patients with abnormal CSF A $\beta$ levels, indicating that, in the presence of $A \beta$ pathology, associations between cerebrovascular, neurodegenerative, and neuroinflammatory processes may be aggravated and contribute to tau aggregation, leading to cognitive impairment and disease progression [71]. Therefore, focusing on these biomarkers offers potential targets for novel therapeutic interventions. In the present work, the administration of Biobran significantly inhibited the levels of ICAM- 1 and IL- 6 in the hippocampi of SAD-induced mice with significant accumulation of amyloid plaques.
In the present study, Biobran exerted an antiapoptotic effect against STZ in a dose-dependent manner. This effect could be due to suppression of cleaved caspase- 3 as well as the proapoptotic protein Bax and through the downregulation of the antiapoptotic protein Bcl-2. It has been suggested previously that $A \beta$ activates the neuronal apoptotic pathway via its accumulation in the mitochondrial membrane and impairment of mitochondrial function [73]. The membrane of mitochondria becomes permeable during mitochondrial apoptosis, and ROS gets released [74]. Apoptogenic proteins such as cytochrome c can thereby be produced, and proapoptotic factors can be introduced into the cytosol from the mitochondria, ultimately activating procaspases and inducing apoptosis [75]. Neuronal loss can be caused by mitochondrial dysfunction via the regulation of proapoptotic proteins like caspase- 3 and Bax and antiapoptotic proteins like Bcl-2 $[14,15,20]$. The ability of Biobran to exert a protective effect against STZ-induced apoptosis is in accordance with our earlier studies showing that Biobran treatment upregulated Bax expression, activated caspase-3, and downregulated $\mathrm{Bcl}-2$ expression; these well-established molecular events in apoptosis have shown that Biobran can protect against glandular stomach carcinogenesis in rats [49], inhibit hepatocarcinogenesis in rats [50], and enhance fractionated X-ray irradiation's anticancer effects for Ehrlich solid tumor-bearing mice [59].

The effect of Biobran on FOXO protein expression in STZ-injected mice hippocampi was also examined. FOXO proteins have a range of biological functions. They are present throughout the body and are selectively expressed in the nervous system. The complex interaction between signal transduction pathways and FOXO proteins in the presence of oxidative stress can significantly impact apoptosis and autophagy $[18,20,76]$. Under oxidative stress conditions, autophagy can be induced by FOXO proteins along with the promotion of cell survival [64]. STZinjected mice showed significantly higher levels of the FOXO protein expression. Biobran decreased significantly these values in a dose-dependent manner. This demonstrates Biobran's protective effect against FOXO-mediated apoptosis in STZ-treated mice. Recently, it was reported that Biobran/MGN-3 is a promising psychoneuroimmune modulatory agent that could improve the quality of life in healthy old adults [31].

Histopathological analysis using H\&E and Congo red stainings further revealed the protective effect of Biobran against STZ-induced neuronal damage in the cerebral cortex and hippocampal sections of mice. Treatment with Biobran reduced the neuronal toxicity observed in STZ-injected mice, with fewer eosinophilic-stained neurons and more healthy neurons with prominent nuclei. This indicates that Biobran could act as a potential candidate to attenuate neurodegeneration and preserve cognitive functions. Hippocampal sections of Biobran-treated groups also exhibited a dosedependent protective effect against $A \beta$ plaque formation. These positive histological effects of Biobran are in agreement with our behavioral and biochemical assessments. The highest dose of Biobran exerted better protection compared to the low and moderate doses. 


\section{Conclusions}

Biobran exerts a dose-dependent protective effect against sporadic $\mathrm{AD}$. This effect is achieved through the targeting of the Nrf2/ARE antioxidant signaling that modulates amyloidogenesis as well as the $\mathrm{Bcl} 2 / \mathrm{Bax} /$ caspase- 3 pathway. To our knowledge, the present study is the first to investigate the protective effect of Biobran against SAD. Our findings suggest that Biobran's activity is able to reduce $\mathrm{A} \beta$ generation and promotes cognitive function recovery. They may suggest the possible applicability of Biobran in clinical trials of human subjects in the management of SAD.

\section{Data Availability}

The data of the present study including the figures and western blot analysis used to support the findings of this study are included within the article.

\section{Conflicts of Interest}

The authors declare there are no conflicts of interest.

\section{Authors' Contributions}

M Ghoneum and N El Sayed planned the study and wrote the manuscript. N El Sayed designed and performed the experiments. Both authors approved the manuscript.

\section{Acknowledgments}

Biobran/MGN-3 was provided by Daiwa Pharm. Co., Ltd, Japan. This work was funded by Diawa Pharmaceutical Co., Ltd., Tokyo, Japan; Grant \#T0099108.

\section{References}

[1] A. Nunomura, G. Perry, M. A. Pappolla et al., "Neuronal oxidative stress precedes amyloid-beta deposition in Down syndrome," Journal of Neuropathology and Experimental Neurology, vol. 59, no. 11, pp. 1011-1017, 2000.

[2] M. C. Polidori, "Oxidative stress and risk factors for Alzheimer's disease: clues to prevention and therapy," Journal of Alzheimer's Disease, vol. 6, no. 2, pp. 185-191, 2004.

[3] M. Bélanger, I. Allaman, and P. J. Magistretti, "Brain energy metabolism: focus on astrocyte-neuron metabolic cooperation," Cell Metabolism, vol. 14, no. 6, pp. 724-738, 2011.

[4] M. P. Mattson, "Pathways towards and away from Alzheimer's disease," Nature, vol. 430, no. 7000, pp. 631-639, 2004.

[5] P. Flagmeier, S. De, D. C. Wirthensohn et al., "Ultrasensitive Measurement of $\mathrm{CA}^{2+}$ Influx into Lipid Vesicles Induced by Protein Aggregates," Angewandte Chemie (International Ed. in English), vol. 56, no. 27, pp. 7750-7754, 2017.

[6] K. Kanninen, R. Heikkinen, T. Malm et al., "Intrahippocampal injection of a lentiviral vector expressing Nrf2 improves spatial learning in a mouse model of Alzheimer's disease," Proceedings of the National Academy of Sciences of the United States of America, vol. 106, no. 38, pp. 16505-16510, 2009.

[7] G. Joshi, K. A. Gan, D. A. Johnson, and J. A. Johnson, "Increased Alzheimer's disease-like pathology in the APP/ PS1 $\Delta$ E9 mouse model lacking Nrf2 through modulation of autophagy," Neurobiology of Aging, vol. 36, no. 2, pp. 664679, 2015.

[8] P. Milani, G. Ambrosi, O. Gammoh, F. Blandini, and C. Cereda, "SOD1 and DJ-1 Converge at Nrf2 Pathway: A Clue for Antioxidant Therapeutic Potential in Neurodegeneration," Oxidative Medicine and Cellular Longevity, vol. 2013, Article ID 836760, 12 pages, 2013.

[9] A. Gugliandolo, P. Bramanti, and E. Mazzon, "Role of Vitamin $\mathrm{E}$ in the Treatment of Alzheimer's Disease: Evidence from Animal Models," International Journal of Molecular Sciences, vol. 18, no. 12, p. 2504, 2017.

[10] Y. Tian, W. Wang, L. Xu et al., "Activation of Nrf2/ARE pathway alleviates the cognitive deficits in PS1V97L Tg mouse model of Alzheimer's disease through modulation of oxidative stress," Journal of Neuroscience Research, vol. 97, no. 4, pp. 492-505, 2018.

[11] I. M. Cojocaru, M. Cojocaru, G. Miu, and V. Sapira, "Study of interleukin-6 production in Alzheimer's disease," Romanian Journal of Internal Medicine, vol. 49, no. 1, pp. 55-58, 2011.

[12] Q. Alam, M. Zubair Alam, G. Mushtaq et al., "Inflammatory Process in Alzheimer's and Parkinson's Diseases: Central Role of Cytokines," Current Pharmaceutical Design, vol. 22, no. 5, pp. 541-548, 2016.

[13] R. Pola, A. Flex, E. Gaetani et al., "Intercellular adhesion molecule-1 K469E gene polymorphism and Alzheimer's disease," Neurobiology of Aging, vol. 24, no. 2, pp. 385-387, 2003.

[14] L. H. Broise, A. R. Gottschalk, J. Quintáns, and C. B. Thompson, "Bcl-2 and Bcl-2-related proteins in apoptosis regulation," Current Topics in Microbiology and Immunology, vol. 200, pp. 107-121, 1995.

[15] E. Castre'n, Y. Ohga, M. P. Berzaghi, G. Tzimagiorgis, H. Thoenen, and D. Lindholm, " $b c l-2$ Messenger RNA is localized in neurons of the developing and adult rat brain," Neuroscience, vol. 61, no. 1, pp. 165-177, 1994.

[16] V. M. Renault, P. U. Thekkat, K. L. Hoang et al., "The prolongevity gene FoxO3 is a direct target of the p53 tumor suppressor," Oncogene, vol. 30, no. 29, pp. 3207-3221, 2011.

[17] J. Hou, S. Wang, Y. Chen Shang, Z. Zhong Chong, and K. Maiese, "Erythropoietin employs cell longevity pathways of SIRT1 to foster endothelial vascular integrity during oxidant stress," Current Neurovascular Research, vol. 8, no. 3, pp. 220 235, 2011.

[18] S. Peng, S. Zhao, F. Yan et al., "HDAC2 selectively regulates foxo3a-mediated gene transcription during oxidative stress induced neuronal cell death," The Journal of Neuroscience, vol. 35, no. 3, pp. 1250-1259, 2015.

[19] S. Wang, Z. Zhong Chong, Y. Chen Shang, and K. Maiese, "WISP1 neuroprotection requires FoxO3a post-translational modulation with autoregulatory control of SIRT1," Current Neurovascular Research, vol. 10, no. 1, pp. 54-69, 2013.

[20] C. Charvet, I. Alberti, F. Luciano et al., "Proteolytic regulation of Forkhead transcription factor FOXO3a by caspase-3-like proteases," Oncogene, vol. 22, no. 29, pp. 4557-4568, 2003.

[21] Y. C. Shang, Z. Z. Chong, J. Hou, and K. Maiese, "Wnt1, FoxO3a, and NF- $\kappa \mathrm{B}$ oversee microglial integrity and activation during oxidant stress," Cellular Signalling, vol. 22, no. 9, pp. 1317-1329, 2010.

[22] M. Salkovic-Petrisic, A. Knezovic, S. Hoyer, and P. Riederer, "What have we learned from the streptozotocin-induced animal model of sporadic Alzheimer's disease, about the 
therapeutic strategies in Alzheimer's research," Journal of Neural Transmission (Vienna), vol. 120, no. 1, pp. 233-252, 2013.

[23] P. K. Kamat, A. Kalani, S. Rai, S. K. Tota, A. Kumar, and A. S. Ahmad, "Streptozotocin Intracerebroventricular-Induced Neurotoxicity and Brain Insulin Resistance: a Therapeutic Intervention for Treatment of Sporadic Alzheimer's Disease (sAD)-Like Pathology," Molecular Neurobiology, vol. 53, no. 7, pp. 4548-4562, 2016.

[24] D. Hsu and G. A. Marshall, "Primary and Secondary Prevention Trials in Alzheimer Disease: Looking Back, Moving Forward," Current Alzheimer Research, vol. 14, no. 4, pp. 426440, 2017.

[25] C. Patterson, World Alzheimer Report 2018: The state of the art of dementia research: new frontiers, Alzheimer's Disease International, London, 2018.

[26] M. Ghoneum, "Anti-HIV Activity in Vitro of MGN-3, an Activated Arabinoxylane from Rice Bran," Biochemical and Biophysical Research Communications, vol. 243, no. 1, pp. 2529, 1998.

[27] M. Ghoneum, "From bench to bedside: The growing use of arabinoxylan rice bran (MGN-3/Biobran) in cancer immunotherapy," Austin Immunology, vol. 1, p. 1006, 2016.

[28] E. Noaman, N. K. Badr el-Din, M. A. Bibars, A. A. Abou Mossallam, and M. Ghoneum, "Antioxidant potential by arabinoxylan rice bran, MGN-3/biobran, represents a mechanism for its oncostatic effect against murine solid Ehrlich carcinoma," Cancer Letters, vol. 268, no. 2, pp. 348-359, 2008.

[29] M. Ghoneum and S. Abedi, "Enhancement of natural killer cell activity of aged mice by modified arabinoxylan rice bran (MGN-3/Biobran)," The Journal of Pharmacy and Pharmacology, vol. 56, no. 12, pp. 1581-1588, 2004.

[30] A. F. Elsaid, M. Shaheen, and M. Ghoneum, "Biobran/MGN-3, an arabinoxylan rice bran, enhances NK cell activity in geriatric subjects: A randomized, double-blind, placebo-controlled clinical trial," Experimental and Therapeutic Medicine, vol. 15, no. 3, pp. 2313-2320, 2018.

[31] A. F. Elsaid, R. M. Fahmi, M. Shaheen, and M. Ghoneum, "The enhancing effects of Biobran/MGN-3, an arabinoxylan rice bran, on healthy old adults' health-related quality of life: a randomized, double-blind, placebo-controlled clinical trial," Quality of Life Research, vol. 29, no. 2, pp. 357-367, 2020.

[32] National Institutes of Health, National Institutes of Health Guide for Care and Use of Laboratory Animals, 2011.

[33] K. Tazawa, BioBran/MGN-3 (Rice Bran Arabinoxylan Deritive): Basic and clinical application to integrative medicine, Iyakushuppan Co. Publishers, 2006.

[34] M. Ghoneum and J. Brown, "NK immunorestoration of cancer patients by MGN-3, a modified arabinoxylan rice bran (study of 32 patients followed for up to 4 years)," Wheat and rice in disease prevention and health. Anti-aging medical therapeutics. Vol. III. Klatz R, Goldman R, R. R. Watson, V. Preedy, and S. Zibadi, Eds., pp. 217-226, 1999.

[35] OECD, "Organization for Economic Cooperation and Development (OECD) Guidelines for Testing of Chemicals. Acute Oral Toxic," Acute Toxic Class Method, vol. 423, 2001.

[36] M. A. Pelleymounter, M. Joppa, N. Ling, and A. C. Foster, "Pharmacological evidence supporting a role for central corticotropin-releasing Factor2Receptors in behavioral, but not endocrine, response to environmental stress," The Journal of Pharmacology and Experimental Therapeutics, vol. 302, no. 1, pp. 145-152, 2002.
[37] G. I. Warnock, Study Of The Central Corticotrophin-Releasing Factor System Using The 2- Deoxyglucose Method For Measurement Of Local Cerebral Glucose Utilisation, Dissertation. University of Bath, 2010.

[38] J. Mehla, M. Pahuja, and Y. K. Gupta, "Streptozotocin-Induced Sporadic Alzheimer's Disease: Selection of Appropriate Dose," Journal of Alzheimer's Disease, vol. 33, no. 1, pp. 17-21, 2012.

[39] A. Ennaceur, "One-trial object recognition in rats and mice: Methodological and theoretical issues," Behavioural Brain Research, vol. 215, no. 2, pp. 244-254, 2010.

[40] G. M. Morris, "Spatial Localization Does Not Require the Presence of Local Cues," Learning and Motivation, vol. 260, pp. 239-260, 1981.

[41] K. Yamada, T. Tanaka, T. Mamiya, T. Shiotani, T. Kameyama, and T. Nabeshima, "Improvement by nefiracetam of $\beta$-amyloid-(1-42)-induced learning and memory impairments in rats," British Journal of Pharmacology, vol. 126, no. 1, pp. 235-244, 1999.

[42] E. Beutler, O. Duron, and B. Kelly, "Improved method for the determination of blood glutathione," The Journal of Laboratory and Clinical Medicine, vol. 61, pp. 882-888, 1963.

[43] M. Mihara and M. Uchiyama, "Determination of malonaldehyde precursor in tissues by thiobarbituric acid test," Analytical Biochemistry, vol. 86, no. 1, pp. 271-278, 1978.

[44] O. H. Lowry, N. J. Rosebrough, A. L. Farr, and R. J. Randall, "Protein measurement with the Folin phenol reagent," The Journal of Biological Chemistry, vol. 193, no. 1, pp. 265-275, 1951.

[45] J. D. Banchroft, A. Stevens, and D. R. Turner, Theory and Practice of Histological Techniques, Churchil Livingstone, New York, London, San Francisco, Tokyo, Fourth edition, 1996.

[46] M. Ghoneum, "Enhancement of human natural killer cell activity by modified arabinoxylan from rice bran (MGN-3)," International Journal of Immunotherapy, vol. 14, pp. 89-99, 1998.

[47] A. Pérez-Martínez, J. Valentín, L. Fernández et al., "Arabinoxylan rice bran (MGN-3/Biobran) enhances natural killer cellmediated cytotoxicity against neuroblastoma in vitro and in vivo," Cytotherapy, vol. 17, no. 5, pp. 601-612, 2015.

[48] M. Ghoneum and S. Agrawal, "Activation of Human Monocyte-Derived Dendritic Cells in Vitro by the Biological Response Modifier Arabinoxylan Rice Bran (MGN-3/BIOBRAN)," International Journal of Immunopathology and Pharmacology, vol. 24, no. 4, pp. 941-948, 2011.

[49] N. K. Badr El-Din, S. M. Abdel Fattah, D. Pan, L. Tolentino, and M. Ghoneum, "Chemopreventive Activity of MGN-3/Biobran Against Chemical Induction of Glandular Stomach Carcinogenesis in Rats and Its Apoptotic Effect in Gastric Cancer Cells," Integrative Cancer Therapies, vol. 15, no. 4, pp. NP26-NP34, 2016.

[50] N. K. Badr El-Din, D. A. Ali, R. Othman, S. W. French, and M. Ghoneum, "Chemopreventive role of arabinoxylan rice bran, MGN-3/Biobran, on liver carcinogenesis in rats," Biomedicine \& Pharmacotherapy, vol. 126, p. 110064, 2020.

[51] H. Salama, E. Medhat, M. Shaheen, A. R. N. Zekri, T. Darwish, and M. Ghoneum, "Arabinoxylan rice bran (Biobran) suppresses the viremia level in patients with chronic HCV infection: A randomized trial," International Journal of Immunopathology and Pharmacology, vol. 29, no. 4, pp. 647-653, 2016.

[52] M. Ghoneum, M. Matsuura, and S. Gollapudp, "Modified arabinoxylan rice bran (Mgn-3/Biobran) enhances intracellular 
killing of microbes by human phagocytic Cellsin vitro," International Journal of Immunopathology and Pharmacology, vol. 21, no. 1, pp. 87-95, 2008.

[53] A. M. El Halawany, N. S. E. L. Sayed, H. M. Abdallah, and R. S. E. Dine, "Protective effects of gingerol on streptozotocininduced sporadic Alzheimer's disease: emphasis on inhibition of $\beta$-amyloid, COX-2, alpha-, beta - secretases and APH1a," Scientific Reports, vol. 7, no. 1, p. 2902, 2017.

[54] N. O. Abdel Rasheed, N. S. El Sayed, and A. S. El-Khatib, “Targeting central $\beta 2$ receptors ameliorates streptozotocininduced neuroinflammation via inhibition of glycogen synthase kinase 3 pathway in mice," Progress in NeuroPsychopharmacology \& Biological Psychiatry, vol. 86, pp. 6575, 2018.

[55] A. Sgarbossa, D. Giacomazza, and M. di Carlo, "Ferulic Acid: A Hope for Alzheimer's Disease Therapy from Plants," Nutrients, vol. 7, no. 7, pp. 5764-5782, 2015.

[56] M. Khalili and F. Hamzeh, "Effects of active constituents of Crocus sativus L., crocin on streptozocin-induced model of sporadic Alzheimer's disease in male rats," Iranian Biomedical Journal, vol. 14, no. 1-2, pp. 59-65, 2010.

[57] P. Liu, L. B. Zou, L. H. Wang et al., "Xanthoceraside attenuates tau hyperphosphorylation and cognitive deficits in intracerebroventricular- streptozotocin injected rats," Psychopharmacologia, vol. 231, pp. 345-356, 2014.

[58] S. Bhatt, L. Puli, and C. R. Patil, "Role of reactive oxygen species in the progression of Alzheimer's disease," Drug Discovery Today, 2020.

[59] M. Ghoneum, N. K. Badr El-Din, S. M. Abdel Fattah, and L. Tolentino, "Arabinoxylan rice bran (MGN-3/Biobran) provides protection against whole-body $\gamma$-irradiation in mice via restoration of hematopoietic tissues," Journal of Radiation Research, vol. 54, no. 3, pp. 419-429, 2013.

[60] Y. V. Nikitchenko, V. K. Klochkov, N. S. Kavok et al., “Antiaging Effects of Antioxidant Rare-Earth Orthovanadate Nanoparticles in Wistar Rats," Biological Trace Element Research, 2021.

[61] J. Poddar, S. Singh, P. Kumar, S. Bali, S. Gupta, and S. Chakrabarti, "Inhibition of complex I-III activity of brain mitochondria after intracerebroventricular administration of streptozotocin in rats is possibly related to loss of body weight," Heliyon, vol. 6, no. 7, article e04490, 2020.

[62] J. Alam and L. Sharma, "Potential Enzymatic Targets in Alzheimer's: A Comprehensive Review," Current Drug Targets, vol. 20, no. 3, pp. 316-339, 2019.

[63] A. Armada-Moreira, J. I. Gomes, C. C. Pina et al., "Going the Extra (Synaptic) Mile: Excitotoxicity as the Road Toward Neurodegenerative Diseases," Frontiers in Cellular Neuroscience, vol. 14, 2020.

[64] C. A. Massaad, "Neuronal and vascular oxidative stress in Alzheimer's disease," Current Neuropharmacology, 2011.

[65] C. P. Ramsey, C. A. Glass, M. B. Montgomery et al., "Expression of Nrf2 in neurodegenerative diseases," Journal of Neuropathology and Experimental Neurology, vol. 66, no. 1, pp. 7585, 2007.

[66] K. Kanninen, T. M. Malm, H. K. Jyrkkänen et al., "Nuclear factor erythroid 2-related factor 2 protects against beta amyloid," Molecular and Cellular Neurosciences, vol. 39, no. 3, pp. 302313, 2008.

[67] T. W. Kensler, N. Wakabayashi, and S. Biswal, "Cell survival responses to environmental stresses via the Keap1-Nrf2-ARE pathway," Annual Review of Pharmacology and Toxicology, vol. 47, no. 1, pp. 89-116, 2007.

[68] A. I. Rojo, M. Pajares, P. Rada et al., "NRF2 deficiency replicates transcriptomic changes in Alzheimer's patients and worsens APP and TAU pathology," Redox Biology, vol. 13, pp. 444-451, 2017.

[69] M. J. Calkins, D. A. Johnson, J. A. Townsend et al., "The Nrf2/ARE pathway as a potential therapeutic target in neurodegenerative disease," Antioxidants \& Redox Signaling, vol. 11, no. 3, pp. 497-508, 2009.

[70] X. Wang, M. R. Campbell, S. E. Lacher et al., "A Polymorphic Antioxidant Response Element Links NRF2/sMAF Binding to Enhanced MAPT Expression and Reduced Risk of Parkinsonian Disorders," Cell Reports, vol. 15, no. 4, pp. 830-842, 2016.

[71] S. Janelidze, N. Mattsson, E. Stomrud et al., "CSF biomarkers of neuroinflammation and cerebrovascular dysfunction in early Alzheimer disease," Neurology, vol. 91, no. 9, pp. e867e877, 2018.

[72] I. Morales, G. Farías, and R. B. Maccioni, "Neuroimmunomodulation in the pathogenesis of Alzheimer's disease," Neuroimmunomodulation, vol. 17, no. 3, pp. 202-204, 2010.

[73] M. Y. Cha, S. H. Han, S. M. Son et al., "Mitochondria-Specific Accumulation of Amyloid $\beta$ Induces Mitochondrial Dysfunction Leading to Apoptotic Cell Death," PLoS One, vol. 7, no. 4, article e34929, 2012.

[74] Z. C. Zhao, F. Li, and K. Maiese, "Stress in the brain: novel cellular mechanisms of injury linked to Alzheimer's disease," Brain Research. Brain Research Reviews, vol. 49, pp. 1-21, 2005.

[75] W. Ahmad, B. Ijaz, K. Shabbiri, F. Ahmed, and S. Rehman, "Oxidative toxicity in diabetes and Alzheimer's disease: mechanisms behind ROS/ RNS generation," Journal of Biomedical Science, vol. 24, no. 1, p. 76, 2017.

[76] K. Maiese, "FoxO proteins in the nervous system," Analytical Cellular Pathology (Amsterdam), vol. 2015, article 569392, 15 pages, 2015. 